\title{
Rapid Progression of Symptomatic Vertebrobasilar Artery Dissection on Magnetic Resonance Imaging: A Case Report
}

\author{
Hyung-Seok Kim, MD', Hyo-Sung Kwak, MD², Seung Bae Hwang, MD², Gyung Ho Chung, MD²
}

Spontaneous intracranial vertebrobasilar dissection can manifest with various clinical symptoms, including subarachnoid hemorrhage or ischemic symptoms from impaired posterior circulation. A 29year-old woman came to our emergency department with a sudden onset of left sided mild motor weakness and headache. Initial magnetic resonance imaging (MRI) showed mild luminal irregularities in the vertebrobasilar arteries with an eccentric periluminal hematoma. Follow-up MRI obtained 3 days later showed a progression of vertebrobasilar dissection to multifocal stenoses with an increased intramural hematoma.

Key Words : Stroke; Vertebrobasilar dissection; Magnetic resonance imaging

Spontaneous intracranial vertebrobasilar dissection (VBD) can manifest as various clinical symptoms in young adults, including subarachnoid hemorrhage, ischemic symptoms from impaired posterior circulation, or localized neurologic symptoms such as headache $[1,2]$. In East Asian populations, intracranial VBD is as common as cervical artery dissection $[2,3]$. The clinical course and prognostic factors for intracranial VBD have been well elucidated [2, 4-6]. However, a rapid progression of intracranial VBD detected by imaging studies has not been reported in the English

'Department of Neurosurgery, IS Hallym Medical Center, Incheon, Korea

${ }^{2}$ Department of Radiology, Chonbuk National University Medical School and Hospital, Jeonju, Korea

Received April 4, 2014; accepted after revision May 29, 2014.

Correspondence to: Hyung-Seok Kim, MD, Department of Neurosurgery, IS Hallym Medical Center, 722, Jangje-ro, Gyeyanggu, Incheon 407-060, Korea.

Tel. 82.32.550.9647 Fax. 82.32.545.1844

E-mail: khs501@hanmail.net

This is an Open Access article distributed under the terms of the Creative Commons Attribution Non-Commercial License (http://creativecommons.org/licenses/by-nc/3.0) which permits unrestricted non-commercial use, distribution, and reproduction in any medium, provided the original work is properly cited. literature. Herein, we report the case of a young female patient with rapid progression of intracranial VBD revealed by serial MRI studies.

\section{CASE REPORT}

A 29-year-old woman came to our emergency department with sudden onset of left sided mild motor weakness and headache. She complained that her headache was pulsatile in the right temporal region, not associated with nausea or vomiting, and developed 14 days prior to the onset of left-sided motor weakness. On admission, her initial blood pressure was 170/90 $\mathrm{mmHg}$. She did not have any past history of head trauma, smoking, diabetes, or cardiovascular disease. An initial head computed tomography (CT) scan did not show any evidence of the intracranial or subarachnoid hemorrhage. MRI was performed to find evidence of cerebral infarction. Diffusion-weighted imaging (DWI) showed a right-sided, acute pontine infarction (Fig. 1A). Time-of-flight (TOF) magnetic resonance angiography (MRA) showed luminal irregularities with mild stenosis of the vertebrobasilar artery (Fig. 1B), but the source image of TOF MRA showed a long-segmen- 


\section{Vertebrobasilar Artery Dissection}

tal, periluminal hematoma (Fig. 1C). Based on these findings, we diagnosed the patient as having extensive VBD with an intramural hematoma.

After 3 days, we performed high-resolution MRI for an evaluation of the extent and severity of VBD. TOF
MRA showed multifocal severe stenoses without dilatation of the vertebrobasilar artery (Fig. 1D). On T1-weighted MRI, the eccentric intramural hematoma increased, involving the longer segment of the vertebrobasilar artery, when compared to the initial
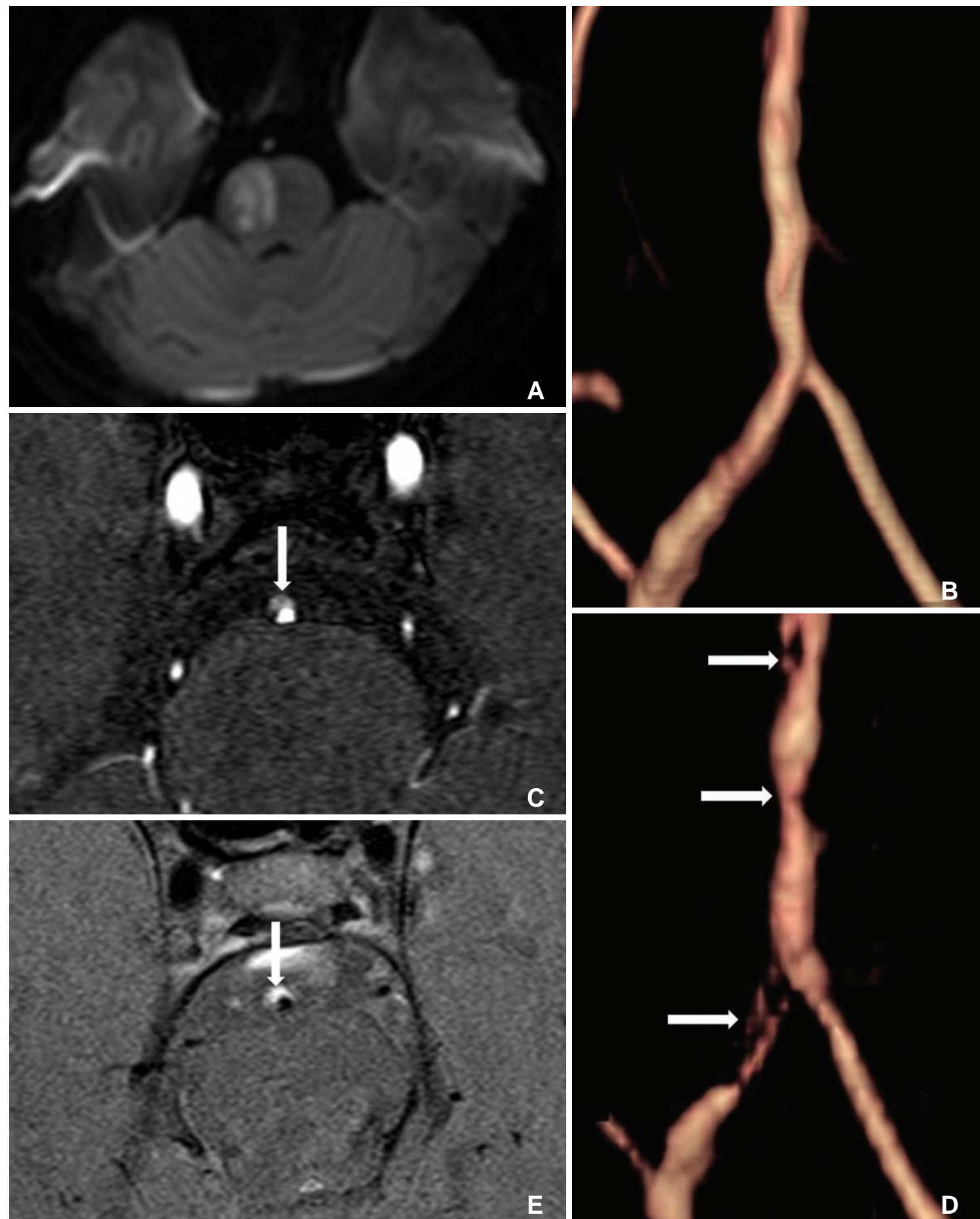

Fig. 1. A. The initial diffusion-weighted imaging showing an acute infarction in the right sided ventral pons. B. Time-of-flight magnetic resonance angiography showing the mild luminal irregularities in the vertebrobasilar arteries. C. A source image of time-of-flight magnetic resonance angiography showing the long-segment periluminal hematoma in the vertebrobasilar artery (arrow). D. Time-of-flight magnetic resonance angiography obtained 3 days later showing the multifocal stenoses (arrows) in the right vertebral and basilar artery. E. Axial, T1-weighted magnetic resonance imaging showing the eccentric intramural hematoma in the vertebrobasilar artery (arrow). 


\section{Hyung-Seok Kim, et al.}

MRI study (Fig. 1E). However, a follow up DWI did not show any new ischemic lesion and her symptoms were well controlled by medical treatment with an antiplatelet agent and anticoagulant during her hospitalization. She was discharged with a stable condition, and no aggravating signs of neurologic symptoms were observed during the last 5 months of follow-up.

\section{DISCUSSION}

Spontaneous dissection of the vertebral artery is a well-recognized cause of ischemic stroke in the vertebrobasilar circulation territory in young and middle-aged adults $[2,5]$. Spontaneous intracranial VBD can manifest with various clinical symptoms, including subarachnoid hemorrhage, ischemic symptoms from impaired posterior circulation, or even local symptoms such as occipital headache and/or neck pain in young adults $[1,2,8,9]$.

Previous studies suggested angiographic findings of the VBDs: tapered narrowing or occlusion, aneurysmal dilatation, intimal flap, retention of contrast agent in the false lumen, and pearl-and-string sign $[1,2,7,8]$. Pathognomonic findings of VBD on MRI are intramural hematoma or intimal flap. In contrast, the pearl-and-string sign is not considered a pathognomonic sign, but is rather a reliable finding [1]. Intramural hematoma was most frequently observed in the pattern of stenosis without dilatation, followed by the pearl-and-string pattern [8].

These changes might be caused by local factors such as an increased intramural hematoma or extended intimal flap. Hemodynamic stress may also play an important role in repeated dissection, resulting in chronically enlarging dissecting aneurysms with multilayered intramural hematomas in some cases and extensive damage to the internal elastic lamina [8]. A systemic factor, such as increased blood pressure might aggravate the natural course of the VBD.
Both MRA and CT angiography offer potential advantages for noninvasive assessment of vascular disease, and they have been previously shown useful in the detection of VBD. Sequential neuroimaging examinations can indicate recanalization or normalization of the blood flow and thus are helpful for the decision to discontinue antithrombotic therapy, but the appropriate timing for follow-up examinations has not yet been defined [5]. In this case report, we showed that MRI and MRA are useful in evaluating an asymptomatic rapid progression of intracranial VBD.

Our case suggests that intracranial VBD can progress rapidly in a short time period, and those changes can be detected with MRI and MRA successfully.

\section{References}

1. Hosoya T, Adachi M, Yamaguchi K, Haku T, Kayama T, Kato T. Clinical and neuroradiological features of intracranial vertebrobasilar artery dissection. Stroke 1999;30:1083-1090

2. Yamaura A, Ono J, Hirai S. Clinical picture of intracranial nontraumatic dissecting aneurysm. Neuropathology 2000;20:85-90

3. Tsukahara T, Minematsu K. Overview of spontaneous cervicocephalic arterial dissection in Japan. Acta Neurochir Suppl 2010; 107:35-40

4. Schievink WI. Spontaneous dissection of the carotid and vertebral arteries. N Engl J Med 2001;344:898-906

5. Arauz A, Marquez JM, Artigas C, Balderrama J, Orrego H. Recanalization of vertebral artery dissection. Stroke 2010;41:717721

6. Kim BM, Kim SH, Kim DI, Shin YS, Suh SH, Kim DJ, et al. Outcomes and prognostic factors of intracranial unruptured vertebrobasilar artery dissection. Neurology 2011;76:1735-1741

7. Yoshimoto Y, Wakai S. Unruptured intracranial vertebral artery dissection. Clinical course and serial radiographic imagings. Stroke 1997;28:370-374

8. Ahn SS, Kim BM, Suh SH, Kim DJ, Kim DI, Shin YS, et al. Spontaneous symptomatic intracranial vertebrobasilar dissection: initial and follow-up imaging findings. Radiology 2012;264:196202

9. Arnold M, Bousser MG, Fahrni G, Fischer U, Georqiadis D, Gandjour J, et al. Vertebral artery dissection: presenting findings and predictors of outcome. Stroke 2006;37:2499-2503 